Volume 70, Number 2, Pages 924-939 (2021)

DOI: $10.31801 /$ cfsuasmas. 898098

ISSN 1303-5991 E-ISSN 2618-6470

Research Article; Received: March 17, 2021; Accepted: May 24, 2021

\title{
APPROXIMATION BY TRUNCATED LUPAŞ OPERATORS OF MAX-PRODUCT KIND
}

\author{
Mediha ÖRKCÜ ${ }^{1}$, Özge DALMANOĞLU ${ }^{2}$, and Fatma Büssra HATIPOĞLU ${ }^{1}$ \\ ${ }^{1}$ Department of Mathematics, Faculty of Science, Gazi University, Ankara, TURKEY. \\ ${ }^{2}$ Department of Mathematics and Science Education, Faculty of Education, \\ Baskent University, Ankara, TURKEY
}

\begin{abstract}
The goals of the present paper are to introduce truncated Lupas type operators of max-product kind and give an estimation for the degree of approximation with respect to first modulus of continuity function. We prove that this estimate can not be improved; on the other hand, for some subclasses of functions, better degree of approximation is obtained. We also showed the piecewise convexity of the constructed operators on the interval $[0,1]$.
\end{abstract}

\section{INTRODUCTION}

As it takes very important place in the approximation theory, the sequences of positive linear operators of discrete type have been studied by various authors in the last century. One of those operators that we deal with in this paper was constructed by A. Lupaş 23 in 1995. His starting point in this construction was the identity

$$
\frac{1}{(1-a)^{\gamma}}=\sum_{k=0}^{\infty} \frac{(\gamma)_{k}}{k !} a^{k}, \quad|a|<1 .
$$

With the help of this identity, he defined the following sequence of operators which is linear and positive:

$$
L_{n}(f)(x)=(1-a)^{n x} \sum_{k=0}^{\infty} \frac{(n x)_{k}}{k !} a^{k} f\left(\frac{k}{n}\right), \quad x \geq 0
$$

2020 Mathematics Subject Classification. 41A25, 41A29, 41A30.

Keywords. Nonlinear max-product operators, Lupaş Operators, the first modulus of continuity function, the rate of convergence.

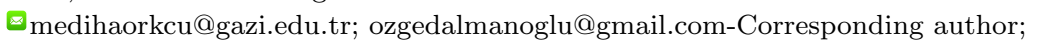
fatmabusrakurt@gmail.com

(D0000-0002-0583-6005; 0000-0002-0322-7265; 0000-0003-0605-0466. 
with $f:[0, \infty) \rightarrow \mathbb{R}$. The notation here is the Pochhammer symbol and given by

$$
(\gamma)_{0}=1, \quad(\gamma)_{k}=\gamma(\gamma+1) \ldots(\gamma+k-1), k \geq 1 .
$$

Imposing $L_{n}\left(e_{1}\right)=e_{1}$, one finds $a=1 / 2$ and therefore the operator turns into,

$$
L_{n}(f)(x)=2^{-n x} \sum_{k=0}^{\infty} \frac{(n x)_{k}}{2^{k} k !} f\left(\frac{k}{n}\right), x \geq 0 .
$$

Agratini [2] studied the approximation properties of these operators by means of Korovkin's theorem and gave estimates for the rate of convergence of the operators. The well-known Korovkin's theorem, which gives a simple proof of Weierstrass theorem, is based on the approximation of functions by linear and positive operators. The underlying algebraic structure of these mentioned operators is linear over $\mathbb{R}$ and they are also linear operators. The idea of nonlinear positive operators was given by Bede et al. in [3. They asked whether they could change the underlying algebraic structure to more general structures. In this sense they presented nonlinear Shepard-type operators by replacing the operations sum and product by max and product.

Following this paper Bede et. al. [4 defined and studied pseudo linear approximation operators. Based upon these studies, there appeared an open problem in the book of S.Gal [17] in which the max-product type Bernstein operators were introduced. Related to this open problem, a nonlinear modification of the classical Bernstein operators were first studied by Bede and Gal [5] in detail. The idea behind these studies were also applied to other well-known approximating operators. Same authors introduced the nonlinear versions of the previously defined operators and they studied the approximation order and shape-preserving properties of the stated operators.

The nonlinear Favard-Szász-Mirakjan operators of max-product type $F_{n}^{(M)}$ is given in [5] as

$$
F_{n}^{(M)}(f)(x)=\frac{\bigvee_{k=0}^{\infty} s_{n, k}(x) f\left(\frac{k}{n}\right)}{\bigvee_{k=0}^{n} s_{n, k}(x)}, x \in[0, \infty), n \in \mathbb{N},
$$

where $s_{n, k}(x)=\frac{(n x)^{k}}{k !}$. Bede, Coroianu and Gal 6. introduced the truncated Favard-Szász-Mirakjan operators of max-product type as follows:

$$
T_{n}^{(M)}(f)(x)=\frac{\bigvee_{k=0}^{n} s_{n, k}(x) f\left(\frac{k}{n}\right)}{\bigvee_{k=0}^{n} s_{n, k}(x)}, x \in[0,1], n \in \mathbb{N} .
$$

Recently, Güngör and İspir studied quantitative estimations for the generalized Szász operators of max product type in 18. Also, they constructed nonlinear 
Bernstein-Chlodowsky operators of max-product type in [19]. Holhos [20] studied weighted approximation of functions by Meyer-König and Zeller operators of max-product type. Coroianu and Gal 8, 9, introduced truncated max-product Kantorovich operators based on Fejer Kernel and generalized $(\varphi, \psi)$-kernels. By Costarelli and Vinti, the max-product neural networks operators were studied in [11- 15]. Recently, in [1], the max-product of Bernstein operators for symmetric ranges are introduced by Acar et.al. and upper estimates of approximation error for some subclasses of functions are obtained. Also, they investigated the shape-preserving properties of the operators.

In this paper, the nonlinear truncated Lupaş operators of max-product type are introduced. We estimate the degree of approximation of the defined sequence of operators. More importantly, we show that the estimate with respect to the modulus of continuity function cannot be improved. On the other hand, for some subclasses of functions, better order of approximation is obtained. Finally, we proved that our sequence of operators is piecewise convex on the interval $[0,1]$ for any arbitrary function $f$.

Before proceeding further, we will recall some general notations about the maxproduct type nonlinear operators. Considering the set of positive real numbers $\mathbb{R}_{+}$, we deal with the maximum " $\bigvee$ " and the product "." operations. Then $\left(\mathbb{R}_{+}, \bigvee, \cdot\right)$ is called as Max-Product algebra.

Let $I \subset \mathbb{R}$ be a bounded or unbounded interval, and

$$
C B_{+}(I)=\left\{f: I \rightarrow \mathbb{R}_{+} ; f \text { continuous and bounded on } I\right\} .
$$

A discrete max-product type approximation operator $L_{n}: C B_{+}(I) \rightarrow C B_{+}(I)$, has a general form

or

$$
L_{n}(f)(x)=\bigvee_{i=0}^{n} D_{n}\left(x, x_{i}\right) \dot{f}\left(x_{i}\right),
$$

$$
L_{n}(f)(x)=\bigvee_{i=0}^{\infty} D_{n}\left(x, x_{i}\right) \dot{f}\left(x_{i}\right)
$$

where $n \in \mathbb{N}, f \in C B_{+}(I), D_{n}(\cdot, x i) \in C B_{+}(I)$ and $x_{i} \in I$, for all $i$. The above form of the operators are positive and nonlinear. These operators also satisfy the pseudolinearity condition which is of the form

$$
L_{n}(a \cdot f \vee b \cdot g)(x)=a \cdot L_{n}(f)(x) \vee b \cdot L_{n}(g)(x), \forall a, b \in \mathbb{R}_{+}, f, g: I \rightarrow \mathbb{R}_{+} .
$$

In order to give some properties of the operators $L_{n}$, we present the following auxiliary Lemma.

Lemma 1. ([5]) Let $L_{n}: C B_{+}(I) \rightarrow C B_{+}(I), n \in \mathbb{N}$ be a sequence of operators satisfying the following properties :

(i) (Monotonicity)

$$
\text { If } f, g \in C B_{+}(I) \text { satisfy } f \leq g \text { then } L_{n}(f) \leq L_{n}(g) \text { for all } n \in \mathbb{N} \text {; }
$$

(ii) (Subadditivity) 
$L_{n}(f+g) \leq L_{n}(f)+L_{n}(g)$ for all $f, g \in C B_{+}(I)$.

Then for all $f, g \in C B_{+}(I), n \in \mathbb{N}$ and $x \in I$ we have

$$
\left|L_{n}(f)(x)-L_{n}(g)(x)\right| \leq L_{n}(|f-g|)(x) .
$$

\section{Construction of the Operators}

Now, we define our truncated max-product type operators as follows:

$$
V_{n}^{(M)}(f)(x)=\frac{\bigvee_{k=0}^{n} v_{n, k}(x) f\left(\frac{k}{n}\right)}{\bigvee_{k=0}^{n} v_{n, k}(x)}, x \in[0,1], n \in \mathbb{N},
$$

where

$$
v_{n, k}(x)=\frac{(n x)_{k}}{2^{k} k !},(n x)_{0}=1, \quad(n x)_{k}=n x(n x+1) \ldots(n x+k-1), k \geq 1 .
$$

We can write the following properties of the operators $V_{n}^{(M)}(f)$.

i) One can see that $\bigvee_{k=0}^{n} v_{n, k}(x)>0$ for $x \in[0,1]$.

For any $f \in C_{+}[0,1]$, the space of all positive real-valued and continuous functions on $[0,1], V_{n}^{(M)}(f) \in C_{+}[0,1]$. So, $V_{n}^{(M)}: C_{+}[0,1] \rightarrow C_{+}[0,1]$ is a sequence of positive operators and since $\bigvee_{k=0}^{n} v_{n, k}(x)=1$ for $x=0, V_{n}^{(M)}(f)(0)=f(0)$.

ii) For any $f \in C_{+}[0,1]$ and $\lambda \geq 0$,

$$
V_{n}^{(M)}(\lambda f)=\lambda V_{n}^{(M)}(f) .
$$

Hence, the max-product operators $V_{n}^{(M)}$ given by 22) are positive homogenous.

iii) For $V_{n}^{(M)}$ the identity

$$
V_{n}^{(M)}\left(e_{0}\right)=e_{0}, e_{0}(x)=1
$$

holds.

iv) $V_{n}^{(M)}(f)$ satisfy the pseudo-linearity condition, i.e., for any $f, g \in C_{+}[0,1]$ and $\alpha, \beta \in \mathbb{R}_{+}$

$$
V_{n}^{(M)}(\alpha f \vee \beta g)(x)=\alpha V_{n}^{(M)}(f)(x) \vee \beta V_{n}^{(M)}(g)(x) .
$$

From the above equality, we have

$$
f \leq g \Longrightarrow V_{n}^{(M)}(f)(x) \leq V_{n}^{(M)}(g)(x) .
$$

So, $V_{n}^{(M)}(f)$ is a monotone operator.

v) For any $f, g \in C_{+}[0,1]$, we get

$$
V_{n}^{(M)}(f+g)(x) \leq V_{n}^{(M)}(f)(x)+V_{n}^{(M)}(g)(x) .
$$


That is the sublinearity condition is satisfied by the operators $V_{n}^{(M)}(f)$.

vi) From the above properties and Lemma 1 we have

$$
\left|V_{n}^{(M)}(f)(x)-V_{n}^{(M)}(g)(x)\right| \leq V_{n}^{(M)}(|f-g|)(x) .
$$

Now, we can write the following corollary.

Corollary 2. For all $f \in C_{+}[0,1]$,

$$
\left|V_{n}^{(M)}(f)(x)-f(x)\right| \leq\left[1+\frac{1}{\delta} V_{n}^{(M)}\left(\varphi_{x}\right)(x)\right] \omega_{1}(f, \delta),
$$

where $\varphi_{x}(t)=|t-x|, t, x \in[0,1]$ and the modulus of continuity function of $f$ is defined as

$$
\omega_{1}(f, \delta)=\max _{\substack{t, x \in[0,1] \\|t-x| \leq \delta}}\{|f(t)-f(x)|\} .
$$

Proof. For the proof, see for example [6].

\section{Auxiliary Lemmas}

In the current section we will give some auxiliary lemmas which we need for the proof of the main theorem.

Lemma 3. Let $x \in\left[\frac{j+1}{n}, \frac{j+2}{n}\right]$ and $j \in\{0,1, \ldots, n-2\}$. Then we have,

$$
\bigvee_{k=0}^{n} v_{n, k}(x)=v_{n, j}(x) \text {. }
$$

Also, $\bigvee_{k=0}^{n} v_{n, k}(x)=1$, for $x \in\left[0, \frac{2}{n}\right]$.

Proof. In fact for fixed $n \in \mathbb{N}$ and $k \geq 0$, the inequality

$$
\begin{aligned}
& 0 \leq v_{n, k+1}(x) \leq v_{n, k}(x) \\
& 0 \leq n x+k \leq 2(k+1)
\end{aligned}
$$

is equivalent to

So, taking $k=0,1, \ldots, n-2$, we get

$$
0 \leq x \leq \frac{k+2}{n}
$$

$$
\begin{aligned}
0 \leq & v_{n, 1}(x) \leq v_{n, 0}(x) \Longleftrightarrow x \in\left[0, \frac{2}{n}\right], \\
0 \leq & v_{n, 2}(x) \leq v_{n, 1}(x) \Longleftrightarrow x \in\left[0, \frac{3}{n}\right], \\
& \ldots \\
0 \leq & v_{n, k+1}(x) \leq v_{n, k}(x) \Longleftrightarrow x \in\left[0, \frac{k+2}{n}\right],
\end{aligned}
$$




$$
0 \leq v_{n, n-1}(x) \leq v_{n, n-2}(x) \Longleftrightarrow x \in[0,1] .
$$

For $k=n-1$ we also have

$$
0 \leq v_{n, n}(x) \leq v_{n, n-1}(x) \Longleftrightarrow x \in\left[0,1+\frac{1}{n}\right] .
$$

From all these inequalities, we can write

$$
x \in\left[0, \frac{2}{n}\right] \Longrightarrow v_{n, k}(x) \leq v_{n, 0}(x)=1 \text { for all } k=0,1, \ldots, n,
$$

also

$$
\begin{aligned}
& x \in\left[\frac{2}{n}, \frac{3}{n}\right] \Longrightarrow v_{n, k}(x) \leq v_{n, 1}(x) \text { for all } k=0,1, \ldots, n, \\
& x \in\left[\frac{3}{n}, \frac{4}{n}\right] \Longrightarrow v_{n, k}(x) \leq v_{n, 2}(x) \text { for all } k=0,1, \ldots, n,
\end{aligned}
$$

in general, for fixed $j=0,1, \ldots, n-2$,

$$
x \in\left[\frac{j+1}{n}, \frac{j+2}{n}\right] \Longrightarrow v_{n, k}(x) \leq v_{n, j}(x) \text { for all } k=0,1, \ldots, n .
$$

So, the proof is completed.

In order to proceed we need the following notations:

For each $k \in\{0,1, \ldots, n\}, j \in\{0,1, \ldots, n-2\}$, state

$$
M_{k, n, j}(x)=\frac{v_{n, k}(x)\left|\frac{k}{n}-x\right|}{v_{n, j}(x)}, m_{k, n, j}(x)=\frac{v_{n, k}(x)}{v_{n, j}(x)} .
$$

It is clear that if $k \geq j+2$ then

$$
M_{k, n, j}(x)=\frac{v_{n, k}(x)\left(\frac{k}{n}-x\right)}{v_{n, j}(x)}
$$

and if $k \leq j$ then

$$
M_{k, n, j}(x)=\frac{v_{n, k}(x)\left(x-\frac{k}{n}\right)}{v_{n, j}(x)} .
$$

Lemma 4. For all $k \in\{0,1, \ldots, n\}, j \in\{0,1, \ldots, n-2\}$ and $x \in\left[\frac{j+1}{n}, \frac{j+2}{n}\right]$, we have

$$
m_{k, n, j} \leq 1 .
$$

Proof. If $k \geq j$ then, since $h(x)=\frac{1}{n x+k}$ is nonincreasing on $x \in\left[\frac{j+1}{n}, \frac{j+2}{n}\right]$, we have

$$
\frac{m_{k, n, j}(x)}{m_{k+1, n, j}(x)}=\frac{2(k+1)}{n x+k} \geq \frac{2(k+1)}{k+j+2} \geq 1 \text {. }
$$


So, $m_{j, n, j}(x) \geq m_{j+1, n, j}(x) \geq \ldots \geq m_{n, n, j}(x)$ is true.

If $k \leq j$ then,

$$
\frac{m_{k, n, j}(x)}{m_{k-1, n, j}(x)}=\frac{n x+k-1}{2 k} \geq \frac{j+k}{2 k} \geq 1,
$$

which implies, $m_{j, n, j}(x) \geq m_{j-1, n, j}(x) \geq \ldots \geq m_{0, n, j}(x)$ is true. Hence for all $k \in\{0,1,2, \ldots, n\}, j \in\{0,1,2, \ldots, n-2\}, n \in \mathbb{N}$ and $x \in\left[\frac{j+1}{n}, \frac{j+2}{n}\right]$, we can write

$$
m_{k, n, j} \leq m_{j, n, j}(x)=1 .
$$

Lemma 5. Let $x \in\left[\frac{j+1}{n}, \frac{j+2}{n}\right]$,

i) If $k \in\{j+2, \ldots, n-2\}$ is such that, $k-\sqrt{3 k+2} \geq j$ then $M_{k, n, j}(x) \geq$ $M_{k+1, n, j}(x)$.

ii) If $k \in\{1,2, \ldots, j\}$ is such that $k+\sqrt{3 k} \leq j$, then $M_{k, n, j}(x) \geq M_{k-1, n, j}(x)$.

Proof. i) Since $g(x)=\frac{1}{n x+k} \frac{k-n x}{k+1-n x}$ is nonincreasing, we can write $g(x) \geq g\left(\frac{j+2}{n}\right)$ and hence get,

$$
\begin{aligned}
\frac{M_{k, n, j}(x)}{M_{k+1, n, j}(x)} & =\frac{2(k+1)}{n x+k} \frac{\frac{k}{n}-x}{\frac{k+1}{n}-x} \\
& \geq \frac{2(k+1)}{k+j+2} \frac{k-j-2}{k-j-1}
\end{aligned}
$$

Then, the condition $k-\sqrt{3 k+2} \geq j$ implies $(k-j)^{2} \geq 3 k-j+2$. This implies $2(k+1)(k-j-2) \geq(k+j+2)(k-j-1)$. So, we have

$$
\frac{M_{k, n, j}(x)}{M_{k+1, n, j}(x)} \geq 1 \text {. }
$$

ii) Since $h(x)=(n x+k-1) \frac{x-\frac{k}{n}}{x-\frac{k-1}{n}}$ is nondecreasing, we can write $g(x) \geq$ $g\left(\frac{j+1}{n}\right)$ and hence get,

$$
\begin{aligned}
\frac{M_{k, n, j}(x)}{M_{k-1, n, j}(x)} & =\frac{(n x+k-1)}{2 k} \frac{x-\frac{k}{n}}{x-\frac{k-1}{n}} \\
& \geq \frac{j+k}{2 k} \frac{j-k+1}{j-k+2} .
\end{aligned}
$$

Then, the condition $k+\sqrt{3 k} \leq j$ implies $(j-k)^{2} \geq 3 k-j$. Since this implies $(j+k)(j-k+1) \geq 2 k(j-k+2)$, we have

$$
\frac{M_{k, n, j}(x)}{M_{k-1, n, j}(x)} \geq 1 \text {. }
$$

So, the proof is completed. 


\section{Degree of Approximation By $V_{n}^{(M)}(f)$}

Our aim is to estimate the degree of convergence of the sequence of max-product operators $V_{n}^{(M)}(f)$ given by 22 with respect to modulus of continuity function and then show that this estimate can not be improved.

Theorem 6. Let $V_{n}^{(M)}(f), n \in \mathbb{N}$ be defined by 2). For all $f \in C_{+}[0,1]$, the following inequality

$$
\left|V_{n}^{(M)}(f)(x)-f(x)\right| \leq 8 \omega_{1}\left(f, \frac{1}{\sqrt{n}}\right), x \in[0,1]
$$

holds.

Proof. One can see from Corollary 2 that, in order to reach the desired inequality, we have to estimate the term

$$
V_{n}^{(M)}\left(\varphi_{x}\right)(x)=\frac{\bigvee_{k=0}^{n} v_{n, k}(x)\left|\frac{k}{n}-x\right|}{\bigvee_{k=0}^{n} v_{n, k}(x)}, x \in[0,1] .
$$

From Lemma 3 we can write, for $x \in\left[0, \frac{2}{n}\right]$,

$$
V_{n}^{(M)}\left(\varphi_{x}\right)(x)=\bigvee_{k=0}^{n} v_{n, k}(x)\left|\frac{k}{n}-x\right|=\max _{k=0,1, \ldots, n}\left\{M_{k, n, 0}(x)\right\}
$$

where $M_{k, n, 0}(x)$ is defined by $(10)$.

If $k=0$ then,

$$
M_{0, n, 0}(x)=x \leq \frac{2}{n}, x \in\left[0, \frac{2}{n}\right]
$$

If $k \geq 1$ then,

for $x \in\left[0, \frac{1}{n}\right]$, since $(1)_{k}=k !$ and $k \leq 2^{k}$,

$$
M_{k, n, 0}(x) \leq \frac{(n x)_{k}}{2^{k} k !} \frac{k}{n} \leq \frac{k}{2^{k} n} \leq \frac{1}{n},
$$

for $x \in\left[\frac{1}{n}, \frac{2}{n}\right]$ and $k=1$,

$$
M_{1, n, 0}(x)=\frac{(n x)_{1}}{2^{1} 1 !}\left(x-\frac{1}{n}\right) \leq \frac{1}{n},
$$

for $x \in\left[\frac{1}{n}, \frac{2}{n}\right]$ and $k=2,3, \ldots, n$

$$
M_{k, n, 0}(x) \leq \frac{(n x)_{k}}{2^{k} k !}\left(\frac{k}{n}-x\right) \leq \frac{(k+1)(k-1)}{2^{k} n} \leq \frac{k^{2}}{2^{k} n} .
$$

If we take $g(x)=\frac{x^{2}}{2^{x}}$, since $g(x) \leq g\left(\frac{2}{\ln 2}\right)<2, x \in[0, \infty)$, we can write

$$
M_{k, n, 0}(x) \leq \frac{k^{2}}{2^{k} n} \leq \frac{2}{n} \text {. }
$$


Consequently, for $x \in\left[0, \frac{2}{n}\right]$

$$
V_{n}^{(M)}\left(\varphi_{x}\right)(x)=\max _{k=0,1, \ldots, n}\left\{M_{k, n, 0}(x)\right\} \leq \frac{2}{n} .
$$

Considering Lemma 3 once more, we can write

$$
V_{n}^{(M)}\left(\varphi_{x}\right)(x)=\max _{k=0,1, \ldots, n}\left\{M_{k, n, j}(x)\right\}, j \in\{1, \ldots, n-2\} .
$$

Now, we will try to obtain an upper estimate for $M_{k, n, j}(x)$ with $x \in\left[\frac{j+1}{n}, \frac{j+2}{n}\right]$, $j \in\{1, \ldots, n-2\}$. We consider the following 3 cases:

i) If $k=j+1$; for $x \in\left[\frac{j+1}{n}, \frac{j+2}{n}\right], M_{j+1, n, j}(x)=\frac{(n x+j)}{2 j+2}\left(x-\frac{j+1}{n}\right) \leq \frac{1}{n}$.

ii) If $k \geq j+2$;

a) Firstly, we suppose that $k-\sqrt{3 k+2}<j$. From Lemma 4 , since $m_{k, n, j}(x) \leq 1$, we write

$$
\begin{aligned}
M_{k, n, j}(x) & =m_{k, n, j}(x)\left(\frac{k}{n}-x\right) \leq \frac{k}{n}-x \leq \frac{k}{n}-\frac{j+1}{n} \\
& \leq \frac{(\sqrt{3 k+2}-1)}{n} \leq \frac{\sqrt{3 n+2}}{n} \leq \frac{3}{\sqrt{n}} .
\end{aligned}
$$

b) Now, we suppose that $k-\sqrt{3 k+2} \geq j$. Since $g(x)=x-\sqrt{3 x+2}$ is nondecreasing on $\left[\frac{1}{12}, \infty\right)$ and since in this case $k \geq j+2$, we take $3 \leq k \leq n$. Since $g$ is nondecreasing, there exists $\bar{k} \in\{3, \ldots, n\}$ of maximum value such that $\bar{k}-\sqrt{3 \bar{k}+2}<$ $j$ and $\bar{k}+1-\sqrt{3 \bar{k}+5} \geq j$.

$$
\begin{aligned}
M_{\bar{k}+1, n, j}(x) & =m_{\bar{k}+1, n, j}(x)\left(\frac{\bar{k}+1}{n}-x\right) \leq \frac{\bar{k}+1}{n}-x \\
& \leq \frac{\bar{k}+1}{n}-\frac{j+1}{n}<\frac{\sqrt{3 \bar{k}+2}}{n} \leq \frac{3}{\sqrt{n}} .
\end{aligned}
$$

So, from Lemma 5 , for $k \in\{\bar{k}+1, \bar{k}+2, \ldots, n\}$, we get $M_{\bar{k}+1, n, j}(x) \geq M_{\bar{k}+2, n, j}(x) \geq$ $\ldots \geq M_{n, n, j}(x)$. Finally, by $\left[13\right.$, we can write $M_{k, n, j}(x) \leq \frac{3}{\sqrt{n}}$, for $k \in\{\bar{k}+1, \bar{k}+2, \ldots, n\}$. iii) $k \leq j$;

a) Firstly, we suppose that $k+\sqrt{3 k}>j$. From Lemma 4 we get

$$
\begin{aligned}
M_{k, n, j}(x) & =m_{k, n, j}(x)\left(x-\frac{k}{n}\right) \leq \frac{j+2}{n}-\frac{k}{n} \\
& \leq \frac{\sqrt{3 k}+2}{n} \leq \frac{\sqrt{3 n}+2}{n} \leq \frac{4}{\sqrt{n}} .
\end{aligned}
$$

b) Now, we suppose that $k+\sqrt{3 k} \leq j$. Since $h(x)=x+\sqrt{3 x}$ is increasing on $[0, \infty)$, there exists $\tilde{k} \in\{1, \ldots, n\}$ of minimum value such that $\tilde{k}+\sqrt{3 \tilde{k}}>j$ and 
$\tilde{k}-1+\sqrt{3 \tilde{k}-3} \leq j$ and

$$
\begin{aligned}
M_{\tilde{k}-1, n, j}(x) & =m_{\tilde{k}-1, n, j}(x)\left(x-\frac{\tilde{k}-1}{n}\right) \leq x-\frac{\tilde{k}-1}{n} \\
& \leq \frac{j+2}{n}-\frac{\tilde{k}-1}{n}<\frac{\tilde{k}+\sqrt{3 \tilde{k}}+2}{n}-\frac{\tilde{k}-1}{n} \\
& =\frac{\sqrt{3 \tilde{k}}+3}{n} \leq \frac{\sqrt{3 n}+2}{n} \leq \frac{4}{\sqrt{n}} .
\end{aligned}
$$

We have $j \geq 1$ in this case. Also, since $j+\sqrt{3 j}>j$ and $\tilde{k} \in\{0,1, \ldots, n\}$ of minimum value such that $\tilde{k}+\sqrt{3 \tilde{k}}>j, \tilde{k}-1 \leq j$. So, from Lemma 5 , for $k \in\{1,2, \ldots, \tilde{k}-1\}$, we get $M_{\tilde{k}-1, n, j}(x) \geq M_{\tilde{k}-2, n, j}(x) \geq \ldots \geq M_{0, n, j}(x)$. Finally, by (14), we can write $M_{k, n, j}(x) \leq \frac{4}{\sqrt{n}}$, for $k \in\{1,2, \ldots, \tilde{k}-1\}$.

Considering all the estimates above, we can write

$$
M_{k, n, j}(x) \leq \frac{4}{\sqrt{n}} \text { for all } x \in\left[\frac{j+1}{n}, \frac{j+2}{n}\right]
$$

which implies

$$
V_{n}^{(M)}\left(\varphi_{x}\right)(x) \leq \frac{4}{\sqrt{n}} \text { for all } x \in[0,1]
$$

Since the first modulus of continuity function satisfies the property $\omega_{1}(f, m \delta) \leq$ $m \omega_{1}(f, \delta)$, for $m \in \mathbb{N}$, with all cases and subcases and taking $\delta=\frac{4}{\sqrt{n}}$ in $[9]$, the proof is completed.

Remark 7. The estimate regarding the first order modulus of continuity function given in Theorem 6 cannot be improved for $n \geq 4$. Suppose that

$$
j_{n}=\left[\frac{n}{2}\right], k_{n}=j_{n}+[\sqrt{n}], x_{n}=\frac{j_{n}}{n},
$$

With calculations, we can write

$$
\begin{aligned}
M_{k_{n}, n, j_{n}}\left(x_{n}\right) & =\frac{\left(n x_{n}+j_{n}\right) \ldots\left(n x_{n}+k_{n}-1\right)}{2^{k_{n}-j_{n}}\left(j_{n}+1\right) \ldots k_{n}}\left|\frac{k_{n}}{n}-x_{n}\right| \\
& \geq \frac{\left(n x_{n}+j_{n}\right)^{k_{n}-j_{n}}}{2^{k_{n}-j_{n}} k_{n}^{k_{n}-j_{n}}}\left|\frac{k_{n}}{n}-x_{n}\right| \\
& =\frac{\left(2 j_{n}\right)^{k_{n}-j_{n}}}{2^{k_{n}-j_{n}} k_{n}^{k_{n}-j_{n}}}\left|\frac{k_{n}}{n}-x_{n}\right| \\
& =\frac{\left(j_{n}\right)^{k_{n}-j_{n}}}{k_{n}^{k_{n}-j_{n}}} \frac{\left(k_{n}-j_{n}\right)}{n}
\end{aligned}
$$




$$
=\left(\frac{\left[\frac{n}{2}\right]}{\left[\frac{n}{2}\right]+[\sqrt{n}]}\right)^{[\sqrt{n}]} \frac{[\sqrt{n}]}{n} .
$$

From definition of the greatest integer function, we can write

$$
\left(\frac{\frac{n}{2}-1}{\frac{n}{2}+\sqrt{n}}\right)^{\sqrt{n}} \leq\left(\frac{\left[\frac{n}{2}\right]}{\left[\frac{n}{2}\right]+[\sqrt{n}]}\right)^{[\sqrt{n}]} \leq\left(\frac{\frac{n}{2}}{\frac{n}{2}+\sqrt{n}-2}\right)^{\sqrt{n}-1}
$$

One can obtain that $\lim _{n \rightarrow \infty}\left(\frac{\left[\frac{n}{2}\right]}{\left[\frac{n}{2}\right]+[\sqrt{n}]}\right)^{[\sqrt{n}]}=e^{-2}$ and there exists $n_{0} \in \mathbb{N}$ such that

$$
M_{k_{n}, n, j_{n}}\left(x_{n}\right) \geq \frac{[\sqrt{n}]}{e^{3} n}, n \geq n_{0}
$$

Also $\frac{[\sqrt{n}]}{\sqrt{n}} \geq \frac{\sqrt{n}-1}{\sqrt{n}} \geq \frac{1}{2}$ for $n \geq 4$. Therefore we can say

$$
M_{k_{n}, n, j_{n}}\left(x_{n}\right) \geq \frac{1}{2 e^{3} \sqrt{n}}, n \geq n_{0} .
$$

Now, we will show that better order of approximation can be obtained for some subclasses of functions $f$.

For $x=0$, since $v_{n, k}(0)=0$ for all $k \in\{1, \ldots, n\}$ and $v_{n, 0}(0)=1, V_{n}^{(M)}(f)(0)-$ $f(0)=0$. So, we assume $x>0$.

For any $k \in 0,1, \ldots n$ and $j \in\{0,1, \ldots, n-2\}$ consider the functions

$$
f_{k, n, j}(x)=\frac{v_{n, k}(x)}{v_{n, j}(x)} f\left(\frac{k}{n}\right)=m_{k, n, j}(x) f\left(\frac{k}{n}\right) .
$$

For any $j \in\{0,1, \ldots, n-2\}$ and $x \in\left[\frac{j+1}{n}, \frac{j+2}{n}\right]$ we can write

$$
V_{n}^{(M)}(f)(x)=\bigvee_{k=0}^{n} \frac{v_{n, k}(x)}{v_{n, j}(x)} f\left(\frac{k}{n}\right)=\bigvee_{k=0}^{n} f_{k, n, j}(x) .
$$

Lemma 8. For $f:[0,1] \longrightarrow[0, \infty)$ and $j \in\{0,1, \ldots, n-2\}$, if

$V_{n}^{(M)}(f)(x)=\max \left\{f_{j, n, j}(x), f_{j+1, n, j}(x), f_{j+2, n, j}(x)\right\}$, for all $x \in\left[\frac{j+1}{n}, \frac{j+2}{n}\right]$

then

$$
\left|V_{n}^{(M)}(f)(x)-f(x)\right| \leq 2 \omega_{1}\left(f, \frac{1}{n}\right), \text { for all } x \in\left[\frac{j+1}{n}, \frac{j+2}{n}\right] .
$$

Proof. We proceed in the same manner as Bede et.al. 66. This time we have three cases to examine: 
Case (i) Let $V_{n}^{(M)}(f)(x)=f_{j, n, j}(x)=f\left(\frac{j}{n}\right)$ for fixed $x \in\left[\frac{j+1}{n}, \frac{j+2}{n}\right]$. Since $\frac{1}{n} \leq x-\frac{j}{n} \leq \frac{2}{n}$

$$
\left|V_{n}^{(M)}(f)(x)-f(x)\right| \leq \omega_{1}\left(f, \frac{2}{n}\right) .
$$

Case (ii) Let $V_{n}^{(M)}(f)(x)=f_{j+1, n, j}(x)$ for fixed $x \in\left[\frac{j+1}{n}, \frac{j+2}{n}\right]$.

Subcase (a) If $V_{n}^{(M)}(f)(x) \leq f(x)$ then

$$
\begin{aligned}
\left|V_{n}^{(M)}(f)(x)-f(x)\right| & =f(x)-f_{j+1, n, j}(x) \\
& \leq f(x)-f_{j, n, j}(x) \leq \omega_{1}\left(f, \frac{2}{n}\right) .
\end{aligned}
$$

Subcase (b) If $V_{n}^{(M)}(f)(x)>f(x)$ then

$$
\begin{aligned}
\left|V_{n}^{(M)}(f)(x)-f(x)\right| & =f_{j+1, n, j}(x)-f(x) \\
& =m_{j+1, n, j}(x) f\left(\frac{j+1}{n}\right)-f(x) \\
& \leq f\left(\frac{j+1}{n}\right)-f(x) .
\end{aligned}
$$

Since $0 \leq x-\frac{j+1}{n} \leq \frac{1}{n}$

$$
\left|V_{n}^{(M)}(f)(x)-f(x)\right| \leq \omega_{1}\left(f, \frac{1}{n}\right) .
$$

Case (iii) Let $V_{n}^{(M)}(f)(x)=f_{j+2, n, j}(x)$ for fixed $x \in\left[\frac{j+1}{n}, \frac{j+2}{n}\right]$.

Subcase (a) If $V_{n}^{(M)}(f)(x) \leq f(x)$ then

$$
\begin{aligned}
\left|V_{n}^{(M)}(f)(x)-f(x)\right| & =f(x)-f_{j+2, n, j}(x) \\
& \leq f(x)-f_{j, n, j}(x) \leq \omega_{1}\left(f, \frac{2}{n}\right) .
\end{aligned}
$$

Subcase (b) If $V_{n}^{(M)}(f)(x)>f(x)$ then

$$
\begin{aligned}
\left|V_{n}^{(M)}(f)(x)-f(x)\right| & =f_{j+2, n, j}(x)-f(x) \\
& =m_{j+2, n, j}(x) f\left(\frac{j+2}{n}\right)-f(x) \\
& \leq f\left(\frac{j+2}{n}\right)-f(x) .
\end{aligned}
$$

Since $0 \leq \frac{j+2}{n}-x \leq \frac{1}{n}$

$$
\left|V_{n}^{(M)}(f)(x)-f(x)\right| \leq \omega_{1}\left(f, \frac{1}{n}\right) .
$$


So the proof is completed.

Theorem 9. For $f:[0,1] \longrightarrow[0, \infty)$ is a nondecreasing function and the function $g:(0,1] \rightarrow[0, \infty) g(x)=\frac{f(x)}{x}$ is nonincreasing, we have

$$
\left|V_{n}^{(M)}(f)(x)-f(x)\right| \leq 2 \omega_{1}\left(f, \frac{1}{n}\right) \text { for all } x \in[0,1] .
$$

Proof. From the monotonocity of $f$ and $k \leq j$,

$$
\begin{aligned}
f_{k-1, n, j}(x) & =\frac{2^{j-k+1} j !}{(k-1) !(n x+k-1) \ldots(n x+j-1)} f\left(\frac{k-1}{n}\right) \\
& \leq \frac{2^{j-k} j !}{k !(n x+k) \ldots(n x+j-1)} \frac{2 k}{(n x+k-1)} f\left(\frac{k}{n}\right) \\
& \leq \frac{2^{j-k} j !}{k !(n x+k) \ldots(n x+j-1)} \frac{2 k}{j+k} f\left(\frac{k}{n}\right) \\
& \leq f_{k, n, j}(x)
\end{aligned}
$$

So, we can write

$$
\begin{aligned}
\bigvee_{k=1}^{j} f_{k, n, j}(x) & =f_{j, n, j}(x), \\
V_{n}^{(M)}(f)(x) & =\bigvee_{k=j}^{n} f_{k, n, j}(x) .
\end{aligned}
$$

Let $k \in\{0,1, \ldots, n\}$ with $k \geq j$. Since $g$ is nonincreasing and $x \in\left[\frac{j+1}{n}, \frac{j+2}{n}\right]$, we have

$$
\begin{aligned}
f_{k+1, n, j}(x) & =\frac{j !(n x+j) \ldots(n x+k)}{2^{k+1-j}(k+1) !} f\left(\frac{k+1}{n}\right) \\
& \leq \frac{j !(n x+j) \ldots(n x+k)}{2^{k+1-j}(k+1) !} \frac{k+1}{k} f\left(\frac{k}{n}\right) \\
& =\frac{j !(n x+j) \ldots(n x+k-1)}{2^{k-j} k !} \frac{(n x+k)}{2 k} f\left(\frac{k}{n}\right) \\
& \leq f_{k, n, j}(x) \frac{k+j+2}{2 k} .
\end{aligned}
$$

So, we get

$$
f_{k+1, n, j}(x) \leq f_{k, n, j}(x), \text { for } k \geq j+2,
$$

from which, we have,

$$
\bigvee_{k=j+2}^{n} f_{k, n, j}(x)=f_{j+2, n, j}(x), j \in\{0,1, \ldots, n-2\} .
$$


From 16 and 17 , we obtain $\sqrt{15}$ for all $x \in\left[\frac{j+1}{n}, \frac{j+2}{n}\right]$, i.e.,

$$
V_{n}^{(M)}(f)(x)=\max \left\{f_{j, n, j}(x), f_{j+1, n, j}(x), f_{j+2, n, j}(x)\right\}, j \in\{0,1, \ldots, n-2\} .
$$

By Lemma 8, the proof is completed.

Lemma 10. [7] If $f:[0,1] \longrightarrow[0, \infty)$ is a concave function then $g:(0,1] \rightarrow[0, \infty)$ $g(x)=\frac{f(x)}{x}$ is nonincreasing.

Proof. For the proof see 7

Corollary 11. For $f:[0,1] \longrightarrow[0, \infty)$ is a nondecreasing concave function, we have

$$
\left|V_{n}^{(M)}(f)(x)-f(x)\right| \leq 2 \omega_{1}\left(f, \frac{1}{n}\right), \text { for all } x \in[0,1] .
$$

Proof. The proof is completed by Theorem 9 and Lemma 10.

The last theorem is about the piecewise convexity of the truncated Lupaş operators of max-product type on the interval $[0,1]$.

Theorem 12. For any $f:[0,1] \longrightarrow \mathbb{R}_{+}, V_{n}^{(M)}(f)$ is convex on $\left[\frac{j+1}{n}, \frac{j+2}{n}\right]$, $j \in\{0,1, \ldots, n-2\}$.

Proof. For any $j \in\{0,1, \ldots, n-2\}$ and $x \in\left[\frac{j+1}{n}, \frac{j+2}{n}\right]$, since we can write $V_{n}^{(M)}(f)(x)=$ $\bigvee_{k=0}^{n} f_{k, n, j}(x)$, we will show for any fixed $j, f_{k, n, j}$ is convex on $\left[\frac{j+1}{n}, \frac{j+2}{n}\right]$. This imply that $V_{n}^{(M)}(f)$ is convex, as being the maximum of convex functions on $\left[\frac{j+1}{n}, \frac{j+2}{n}\right]$. Since $f \geq 0$ and $f_{k, n, j}(x)=\frac{(n x)_{k} j !}{2^{k-j}(n x)_{j} k !} f\left(\frac{k}{n}\right)$, we will prove that $g_{k, n, j}(x)=\frac{(n x)_{k}}{(n x)_{j}}$ are convex on $\left[\frac{j+1}{n}, \frac{j+2}{n}\right]$.

For $k=j, g_{j, n, j}$ is constant so it is convex.

For $k=j+1, g_{j+1, n, j}(x)=n x+j$ is convex.

For $k=j-1, g_{j-1, n, j}(x)=\frac{1}{n x+j-1}$ and since $g_{j-1, n, j}^{\prime \prime}(x)=\frac{2 n^{2}}{(n x+j-1)^{3}}>0$ on $\left[\frac{j+1}{n}, \frac{j+2}{n}\right]$, it is convex.

For $k \geq j+2, g_{k, n, j}(x)=(n x+j) \ldots(n x+k-1)$ and $\ln \left(g_{k, n, j}(x)\right)=\ln (n x+j)+$ $\ldots+\ln (n x+k-1)$. Since $g_{k, n, j}^{\prime}(x)=n g_{k, n, j}(x)\left[\frac{1}{n x+j}+\ldots+\frac{1}{n x+k-1}\right]$ and $g_{k, n, j}^{\prime \prime}(x)=n g_{k, n, j}^{\prime}(x)\left[\frac{1}{n x+j}+\ldots+\frac{1}{n x+k-1}\right]-n^{2} g_{k, n, j}(x)\left[\frac{1}{(n x+j)^{2}}+\ldots+\frac{1}{(n x+k-1)^{2}}\right]$, we obtain

$g_{k, n, j}^{\prime \prime}(x)=n^{2} g_{k, n, j}(x)\left\{\left(\frac{1}{n x+j}+\ldots+\frac{1}{n x+k-1}\right)^{2}-\left(\frac{1}{(n x+j)^{2}}+\ldots+\frac{1}{(n x+k-1)^{2}}\right)\right\}>$ 0 .

For $k \leq j-2, g_{k, n, j}(x)=\frac{1}{(n x+k) \ldots(n x+j-1)}$ and since $g_{k, n, j}^{\prime}(x)=-n g_{k, n, j}(x)\left[\frac{1}{n x+k}+\ldots+\frac{1}{n x+j-1}\right]$, we get 
$g_{k, n, j}^{\prime \prime}(x)=n^{2} g_{k, n, j}(x)\left\{\left(\frac{1}{n x+k}+\ldots+\frac{1}{n x+j-1}\right)^{2}+\left(\frac{1}{(n x+k)^{2}}+\ldots+\frac{1}{(n x+j-1)^{2}}\right)\right\}>$ 0 .

Hence, we see that all the functions $g_{k, n, j}$ are convex on $\left[\frac{j+1}{n}, \frac{j+2}{n}\right]$. As being maximum of all these functions, $V_{n}^{(M)}(f)$ is convex on $\left[\frac{j+1}{n}, \frac{j+2}{n}\right]$.

\section{Conclusion}

The nonlinear max product type operators have been studied by various authors for the last two decades. For example, in [16] Duman obtained convergence results for a sequence of max-product operators in the statistical sense. Karakuş and Demirci 22 examined the $\sigma$-statistical convergence of the max product type operators. For the future studies, one can examine whether the truncated Lupaş operators of max product kind can be generalized in the light of these studies or not. Also the statistical convergence of the constructed operators may be investigated.

Another study related to this topic is due to Holhoş. He examined the approximation properties of Meyer-König and Zeller and Favard-Szász-Mirakyan operators of max-product type in weighted space of functions in the papers [20] and [21], respectively. Taking these studies into account, Lupaş operators of max-product type may be constructed on an unbounded interval $[0, \infty)$ and weighted approximation results of the operators can be examined.

Very recently, Coroianu and Gal [10] have studied the Kantorovich type maxproduct operators. In view of this paper one can consider the Lupaş-Kantorovich operators of max-product type and analyze the approximating properties.

Author Contribution Statements M. Örkcü, Ö. Dalmanoğlu and F.B. Hatipoğlu contributed to the design and implementation of the submitted paper, to the analysis of the results and to the writing of the manuscript.

Declaration of Competing Interests We declare that there is no competing interests.

\section{REFERENCES}

[1] Acar, E., Karahan, D., Kirci Serenbay, S., Approximation for the Bernstein operator of max-product kind in symmetric range, Khayyam J. Math., 6 (2) (2020), 257-273. DOI: $10.22034 / \mathrm{kjm} .2020 .109823$

[2] Agratini, O., On a sequence of linear and positive operators, Facta Univ. Ser. Math. Inform., 14 (1999), 41-48.

[3] Bede, B., Nobuhara, H., Fodor, J., Hirota, K., Max-product Shepard approximation operators, Journal of Advanced Computational Intelligence and Intelligent Informatics, 10 (4) (2006), 494-497. DOI:10.20965/jaciii.2006.p0494

[4] Bede, B., Nobuhara, H., Daňková, M., Di Nola, A., Approximation by pseudo- linear operators, Fuzzy Sets and Systems, 159 (2008), 804-820. DOI:10.1016/j.fss.2007.11.007 
[5] Bede, B., Gal, S. G., Approximation by nonlinear Bernstein and Favard-Szász-Mirakjan operators of max-product kind, Journal of Concrete and Applicable Mathematics, 8 (2010), 193-207.

[6] Bede, B., Coroianu, L., Gal, S. G., Approximation by truncated Favard-Szász-Mirakjan operator of max-product kind, Demonstratio Mathematica, 44 (1) (2011), 105-122. DOI:10.1515/dema-2013-0300

[7] Bede, B., Coroianu, L., Gal, S. G., Approximation and shape preserving properties of the Bernstein operator of max-product kind, Int. J. Math. Math. Sci., 590589, (2009). DOI: $10.1155 / 2009 / 590589$

[8] Coroianu, L.,Gal, S. G., $L_{p}$-approximation by truncated max-product sampling operators of Kantorovich-type based on Fejér kernel, Journal of Integral Equations and Applications, 29 (2) (2017), 349-364. DOI: 10.1216/JIE-2017-29-2-349

[9] Coroianu, L., Gal, S. G., Approximation by truncated max-product operators of Kantorovichtype based on generalized $(\varphi, \psi)$-kernels, Mathematical Methods in the Applied Sciences, DOI: $10.1002 /$ mma.5262.

[10] Coroianu, L., Gal, S. G., Approximation by max-product operators of Kantorovich type, Studia Universitatis Babes-Bolyai, Mathematica, 64 (2) (2019), 207-223. DOI:10.24193/subbmath.2019.2.07

[11] Costarelli, D., Vinti, G., Convergence results for a family of Kantorovich max-product neural network operators in a multivariate setting, Math Slovaca, 67 (6) (2017), 1469-1480. DOI:10.1515/ms-2017-0063

[12] Costarelli, D., Vinti, G., Estimates for the neural network operators of the max-product type with continuous and p-integrable functions, Results Math., 73 (12) (2018). DOI: 10.1007/s00025-018-0790-0

[13] Costarelli, D., Vinti, G., Approximation by max-product neural network operators of Kantorovich type, Results Math., 69 (1-2) (2016), 505-519. DOI: 10.1007/s00025-016-0546-7

[14] Costarelli, D., Vinti, G., Saturation classes for max-product neural network operators activated by sigmoidal functions, Results Math, 72 (3), (2017), 1555-1569. DOI: 10.1007/s00025017-0692-6

[15] Costarelli, D., Vinti, G., Max-product neural network and quasiinterpolation operators activated by sigmoidal functions, J. Approx. Theory, 209 (2016), 1-22. DOI: 10.1016/j.jat.2016.05.001

[16] Duman, O., Statistical convergence of max-product approximating operators, Turkish Journal of Mathematics, 34 (4) (2010), 501-514. DOI:10.3906/mat-0807-32

[17] Gal, S. G., Shape-Preserving Approximation by Real and Complex Polynomials, Birkhauser Publ. Co., Boston, Basel, Berlin, 2008. DOI: 10.1007/978-0-8176-4703-2

[18] Güngör, Ş. Y., İspir N., Quantitative estimates for generalized Szász operators of max product kind, Results in Mathematics, 70 (3) (2016), 447-456. DOI: 10.1007/s00025-016-0579-y

[19] Güngör, Ş. Y., İspir N., Approximation by Bernstein-Chlodowsky operators of max-product kind, Mathematical Communications, 23 (2018), 205-225.

[20] Holhos, A., Weighted approximation of functions by Meyer-König and Zeller operators of max-product type, Numerical Functional Analysis and Optimization, 39 (6) (2018), 689-703. DOI: $10.1080 / 01630563.2017 .1413386$

[21] Holhoş, A., Approximation of functions by Favard-Szász-Mirakyan operators of max-product type in weighted spaces, Filomat, 32 (7) (2018), 2567-2576. DOI: 10.2298/FIL1807567H

[22] Karakuş, S., Demirci, K., Statistical $\sigma$ approximation to max-product operators, Computers and Mathematics with Applications, 61 (4) (2011), 1024-1031. DOI:10.1016/j.camwa.2010.12.052

[23] Lupaş, A., The approximation by some positive linear operators, In: Proceedings of the International Dortmund Meeting on Approximation Theory (M.W. Müller et al., eds.), Akademie Verlag, Berlin, (1995), 201-229. 